\title{
EL DISCURSO DEL PROFESORADO DE EDUCACIÓN MUSICAL EN LA INNOVACIÓN EDUCATIVA CON TIC: POSICIONAMIENTOS EN LA EVALUACIÓN DEL SOFTWARE TACTUS
}

\author{
Manuel Jesús Espigares Pinazo \\ Universidad Internacional de la Rioja \\ manueljesus.espigares@unir.net \\ Rafael García Pérez \\ Universidad de Sevilla \\ rafaelgarcia@us.es \\ Jesús Tejada \\ Universidad de Valencia \\ jesustejada@uv.es \\ María Ángeles Rebollo Catalán \\ Universidad de Sevilla \\ rebollo@us.es
}

\begin{abstract}
En este estudio empírico se analizan los posicionamientos del profesorado en torno a la evaluación de un software diseñado ad hoc, para el aprendizaje del ritmo musical denominado "Tactus". El objetivo principal es estudiar los discursos y opiniones del profesorado en la evaluación de dicho software, lo que se articula mediante el análisis de los discursos $(n=115)$ en 8 grupos de discusión, a través de un modelo explicativo de 5 clústeres. El estudio aporta una visión diversa, variada y completa en la evaluación de software educativo a partir de los discursos docentes.
\end{abstract}

This paper presents teachers positioning analysis around the assessment of ad hoc software designed for learning musical rhythm called "Tactus". The main objective is to study the teachers speeches and opinions in software assessment, which is built through the speeches analysis $(n=115)$ in 8 focus groups, with a descriptive model of 5 clusters. The study gives us a diverse, varied and a full picture in educational software evaluation trough teachers' discourses analysis.

\section{INTRODUCCIÓN}

En este artículo se presenta un estudio de los discursos del profesorado que ha evaluado un software educativo para el adiestramiento del ritmo musical. Se centra en el análisis del discurso de ocho grupos de discusión formados por profesorado de instituciones de Educación Musical de España y Chile, que evalúa un software para el aprendizaje del ritmo musical, denominado Tactus. La evaluación de software educativo ya ha sido tratada previamente en otras investigaciones (Konstantidinis, Thrasyvoulos, Theodouli \& Pomporsis, 2010; Reynolds, Walker \& Speight, 2010; Tiernan, 2010; Tudurí, Serra \& Mut, 2013). Los programas educativos ofrecen nuevas posibilidades de formación en las que el alumnado aprende a aprender de una manera más autónoma, sencilla y motivadora. Además, complementa la formación en el aula, dentro de espacios de colaboración y aprendizaje en entornos virtuales.

A nivel teórico, el presente estudio realiza una evaluación de los discursos del profesorado fundamentada en la teoría social y cultural. Las aproximaciones socioculturales que se han hecho al respecto desde el campo de la investigación educativa al análisis del discurso han sido variadas (De Pablos, 1999; Rebollo, 2001; 2002). En este caso, se analizan las distintas valoraciones que se detraen de los discursos sobre el software, que van desde discursos de resistencia al cambio (posiciones tecnófobas) hasta de legitimación del cambio y la innovación (posiciones tecnófilas) en la enseñanza de la música.

En el presente trabajo, se aborda el estudio de las opiniones y discursos docentes en la evaluación de software educativo en el ámbito de las Tecnologías de la Información y la Comunicación (TIC). Para ello, es necesario, tal y como señalan Boza, Tirado \& Guzmán-Franco (2010, p. 2), "explicitar algunas de las creencias que el profesorado tiene sobre el uso de la tecnología en la educación, y con 
ello contribuir a la generación de discursos necesarios que orienten el sentido de la integración tecnológica". Estos discursos revelan mucho acerca del profesorado. El análisis de la implantación de las TIC en el ámbito educativo español ha sido abordado por diversos autores como Boza, Tirado \& Guzmán-Franco (2010). Tratan los factores facilitadores/obstaculizadores de la implantación de las TIC en los procesos de integración tecnológica hallando bastantes obstáculos. Al respecto, Aviram (2002) señala que las escuelas no están todavía suficientemente familiarizadas con las TIC y no tienen una implantación profunda que se haga patente en los procesos de enseñanza-aprendizaje. Añade que el empleo de las TIC no ha conducido a un mayor nivel de los aprendizajes en el alumnado, ni métodos educativos más activos y que "no existe una línea de investigación continuada basada en el discurso consciente que analice las diversas hipótesis, las examine a la luz de los datos disponibles y que intente decidir entre ellas" (2002, p. 10). También señala que "es esencial un proceso sistemático y riguroso de pensamiento estratégico razonado" (2002, p. 4), cuestión que aún no está resuelta en el ámbito educativo relacionado con la implantación de las TIC. En definitiva, parece oportuno continuar con los esfuerzos que pasan tanto por el diseño de los materiales como por la formación del profesorado.

Sin embargo, el desarrollo observable en la incorporación de las TIC en el ámbito educativo no está exento de ciertas limitaciones. Algunos autores identifican obstáculos de distinto orden en el uso de las TIC dentro de los procesos de enseñanza-aprendizaje (Ertmer, 1999; Pelgrum, 2001; Boza, Tirado \& Guzmán-Franco, 2010). Hay barreras denominadas de primer orden, que tienen un carácter externo al profesorado (acceso a la tecnología, disponibilidad de tiempo, apoyos, materiales, formación), existiendo otras de carácter interno (posicionamientos, creencias, bloqueos, resistencias) que afectan a los esfuerzos docentes y a la voluntad del profesorado para integrar la tecnología en el aula (Brickner, 1995). Estos últimos incluyen: su concepción acerca de la instrucción, actitudes hacia las nuevas tecnologías educativas y posicionamientos ante las reformas y los cambios de la práctica docente necesarios para su implantación.

En el estudio de los discursos del profesorado sobre el software Tactus, se abordan las posiciones, opiniones y valoraciones diversas analizando la acción discursiva (Rebollo, 2001). Complementariamente, se cuenta con las contribuciones de Tryantafyllaki (2010), Georgii-Hemming \& Westvall (2010) y Partti \& Karlsen (2010) acerca del estudio de la identidad docente en el campo de la Educación Musical. También se utilizan las nociones teóricas que ofrece la teoría del posicionamiento (en inglés, positioning). Ésta se basa en el concepto de posicionamiento subjetivo que exponen Davies \& Harré (1990), los cuales desarrollan una consideración del pensamiento en términos de "posicionamiento discursivo", o sea, el modo en el que los sujetos adoptan posiciones a lo largo de su discurso. En este sentido, el posicionamiento destaca las relaciones entre el discurso y los contextos intersubjetivos en los que se construyen ideas compartidas.

La importancia de estas teorías para el presente trabajo, radica en que se pueden reconocer de forma detallada los intercambios comunicativos que se producen en el discurso y las posiciones que los sujetos adoptan en sus opiniones sobre el software empleado en el estudio, tal y como se verá más adelante (basado en Harré \& Langenhove, 1999). Además, cuando las personas participan, no sólo expresan su opinión personal sino que además interactúan dentro de un contexto social y comunicativo en el que se produce el intercambio de significados y concepciones del software educativo. Por ello, la denominada teoría del posicionamiento constituye un aparato conceptual y metodológico adecuado para realizar el análisis de las opiniones del profesorado acerca del mismo, dentro de un grupo de discusión como el que se aborda en este trabajo. En éste, se adopta una visión constructivista, social y cultural, según la cual la identidad es una realidad múltiple y negociada constantemente, un proceso narrativo-discursivo por el que las personas, a través de las prácticas sociales y culturales en las que participan, establecen quiénes son en su relación con otras personas y con los contextos y escenarios sociales en general. 
Por otra parte, además de las posiciones que adoptan los sujetos en la valoración de Tactus, la investigación aborda otros aspectos del discurso del profesorado como los planos discursivos o las voces internas, entendidas éstas como el autodiálogo consciente que los sujetos mantienen con sí mismos y su contexto social y cultural, que afectan en sus juicios y valoraciones del software Tactus. Además, las voces que el profesorado alberga en sus discursos pueden ser un instrumento de gran valor para observar las perspectivas, puntos de vista y dimensiones acerca de la evaluación cualitativa de dicho software.

El estudio se apoya en la idea de que las identidades se construyen dentro de un proceso de socialización, en espacios de interacción donde la imagen del docente se configura bajo el reconocimiento del otro (Bolívar, Fernández \& Molina, 2004). Según Bolívar (2007), la identidad como constructo teórico está conformada tanto por los aspectos racionales o cognitivos como por los no racionales o afectivos-emocionales. Estos posicionamientos identitarios condicionan el compromiso, la motivación y la actitud a la hora de afrontar la innovación con TIC en la docencia. La formación de las identidades se produce en la interacción entre el plano individual del sujeto y el plano social, a través de su acción discursiva en su relación con los demás en el ámbito profesional. Autores como Rebollo y Hornillo (2010), indican que la actitud de los sujetos en la evaluación constituye un componente clave en el estudio de la identidad, que muestra la postura individual en situaciones concretas y los significados que se construyen en situaciones compartidas. En esta línea, otros trabajos destacan la importancia de este proceso de construcción de la identidad docente (Alonso et al., 2010), éstos también remarcan la importancia de la identidad y la visión del profesorado en torno a la innovación educativa y las TIC en los procesos de enseñanza-aprendizaje innovadores. A este respecto, Dubar (2002, p. 11) añade que, la identidad es el resultado de una "identificación" contingente, vinculada al proceso socio-histórico, entendida desde la perspectiva de la individualidad en entornos socio-culturales; un proceso de posicionamiento relacional, social y político al mismo tiempo. En conexión con las ideas anteriormente expuestas, Coll \& Falsafi (2010), afirman que cada grupo profesional genera sus propios discursos (lenguajes sociales y géneros discursivos en los términos en los que lo plantea Werstch, 1993), que se vinculan a la creación de la identidad profesional. Por lo tanto, dentro de este proceso de creación y desarrollo de la identidad docente se producen rasgos distintivos e identificativos en los discursos, que definen a nivel social e individual las posiciones del profesorado en un determinado contexto sociocultural.

A partir de las ideas teóricas anteriormente expuestas, que fundamentan el estudio de las opiniones y discursos del profesorado que evalúa el software educativo Tactus, se presenta el apartado metodológico con las categorías establecidas y los rasgos del tipo análisis de dichas valoraciones.

\section{METODOLOGÍA}

La siguiente investigación se plantea desde una metodología fundamentalmente cuantitativa. El objetivo principal es estudiar los discursos y opiniones del profesorado en la evaluación del software Tactus, lo que se articula mediante el análisis de dichas opiniones divididas en distintas categorías, que construye el profesorado en la evaluación de dicho software.

\section{Procedimiento}

En cuanto al procedimiento, se realizan ocho grupos de discusión sobre el software Tactus, en los que el profesorado participa expresando y discutiendo su valoración en torno al uso de Tactus como software educativo integrado en las enseñanzas musicales (ver tabla 1). El profesorado que participa en el estudio es una parte de la plantilla de cada centro educativo. Dicho profesorado (que aparece en la muestra de la investigación) se ha ofrecido voluntariamente para participar en el estudio.

\begin{tabular}{|c|c|c|c|}
\hline $\begin{array}{c}\text { Grupo } \\
\text { de } \\
\text { discusión }\end{array}$ & $\begin{array}{c}\text { Enseñanzas en las que se introduce } \\
\text { Tactus }\end{array}$ & Centro / Lugar & $\begin{array}{c}\text { Número } \\
\text { profesores }\end{array}$ \\
\hline
\end{tabular}




\begin{tabular}{|c|c|c|c|}
\hline 1 & $\begin{array}{l}\text { Enseñanzas elementales: } 1^{\circ} \text { y } 2^{\circ} \text { de } \\
\text { Lenguaje Musical }\end{array}$ & $\begin{array}{l}\text { Escuela de Música de Catarroja } \\
\text { (Valencia) }\end{array}$ & 5 \\
\hline 2 & Licenciatura en Música & Universidad de Serena (Chile) & 6 \\
\hline 3 & $\begin{array}{l}\text { Enseñanzas elementales: } 1^{\circ} \text { y } 2^{\circ} \text { de } \\
\text { Lenguaje Musical }\end{array}$ & Conservatorio de Las Palmas & 4 \\
\hline 4 & $\begin{array}{l}\text { Enseñanzas elementales: } 1^{\circ} \text { y } 2^{\circ} \text { de } \\
\text { Lenguaje Musical }\end{array}$ & Conservatorio Arturo Soria (Madrid) & 7 \\
\hline 5 & Licenciatura en Pedagogía Musical & $\begin{array}{l}\text { Universidad Metropolitana de } \\
\text { Ciencias de la Educación y } \\
\text { Universidad de Chile }\end{array}$ & 6 \\
\hline 6 & $\begin{array}{l}\text { Enseñanzas elementales: } 1^{\circ} \text { y } 2^{\circ} \text { de } \\
\text { Lenguaje Musical }\end{array}$ & $\begin{array}{l}\text { Conservatorio Francisco Guerrero } \\
\text { (Sevilla) }\end{array}$ & 4 \\
\hline 7 & $\begin{array}{l}\text { Enseñanzas elementales: } 1^{\circ} \text { y } 2^{\circ} \text { de } \\
\text { Lenguaje Musical }\end{array}$ & Conservatorio de Tenerife & 8 \\
\hline 8 & $\begin{array}{l}\text { Enseñanzas elementales: } 1^{\circ} \text { y } 2^{\circ} \text { de } \\
\text { Lenguaje Musical }\end{array}$ & $\begin{array}{l}\text { Escola de Música de Rubí } \\
\text { (Barcelona) }\end{array}$ & 5 \\
\hline \multicolumn{3}{|c|}{ Total } & 45 \\
\hline
\end{tabular}

Tabla 1. Grupos de discusión de la investigación

El profesorado ha practicado con Tactus, integrándolo en sus clases durante dos semanas ${ }^{1}$ en el aprendizaje musical del alumnado. En esas dos semanas, se han realizado las sesiones de trabajo con el software y el grupo discusión (al final) que ha sido grabado y transcrito para su posterior estudio. En total ha habido dos sesiones de trabajo con el alumnado. Las sesiones de trabajo han sido de una hora con cada uno de los grupos. Durante la primera sesión de trabajo se ha explicado el funcionamiento básico del software de adiestramiento rítmico y han tenido la oportunidad de usar el programa informático. En la segunda sesión, han seguido utilizando el programa y al final han respondido a un cuestionario acerca de la herramienta para el adiestramiento rítmico (dicho cuestionario no es objeto de estudio en este artículo, por ello no aparece incluido en el mismo). La muestra del alumnado y el profesorado participante en la innovación queda también recogida en la tabla siguiente:

\footnotetext{
${ }^{1}$ Los datos han sido recogidos en los distintos lugares (descritos en la tabla) donde se ha llevado a cabo el estudio a lo largo del año 2009.
} 


\begin{tabular}{|c|c|c|c|c|}
\hline & \multicolumn{2}{|c|}{ Alumnado } & \multicolumn{2}{|c|}{ Profesorado } \\
\hline Centro educativo & $\mathrm{N}\left(\mathrm{h}+\mathrm{m}^{2}\right)$ & Rango edad & $\mathrm{N}(\mathrm{h}+\mathrm{m})$ & Rango edad \\
\hline \multicolumn{5}{|c|}{ Versión preliminar de Tactus (prueba piloto) } \\
\hline 1. Conservatorio de Catarroja (Valencia) & $12(6+6)$ & $9-11$ & $4(2+2)$ & $32-43$ \\
\hline 2. Conservatorio Arturo Soria (Madrid) & $12(5+7)$ & $9-16$ & $6(4+2)$ & $29-39$ \\
\hline 3. Conservatorio Francisco Guerrero (Sevilla) & $52(29+23)$ & $8-11$ & $3(1+2)$ & $41-51$ \\
\hline 4. Conservatorio de Música de Godella (Valencia) & $11(10+1)$ & $9-15$ & $8(6+2)$ & $26-43$ \\
\hline TOTAL & $87(50+37)$ & $9-16$ & $21(13+8)$ & \\
\hline \multicolumn{5}{|c|}{ Versión definitiva de Tactus } \\
\hline 5. U. Chile (Santiago de Chile) & $12(5+7)$ & $9-14$ & $7(3+4)$ & $31-68$ \\
\hline 6. Univ. Metropolitana de CC.EE. (Santiago de Chile) & $9(4+5)^{3}$ & $18-24$ & $4(2+2)$ & $32-43$ \\
\hline 7. ULS (La Serena, Chile) & $\begin{array}{c}24 \\
(10+14)^{4}\end{array}$ & $10-32$ & $5(4+1)$ & $29-36$ \\
\hline 8. Escola de Música de Rubí (Barcelona) & $12(8+4)$ & $10-12$ & $4(0+4)$ & $41-52$ \\
\hline 9. Conservatorio de Tenerife & $12(6+6)$ & $9-11$ & $7(1+6)$ & $41-65$ \\
\hline 10. Conservatorio de Las Palmas de G. C. & $17(6+11)$ & $8-13$ & $3(0+3)$ & $32-58$ \\
\hline 11. Conservatorio de Logroño & $12(6+6)$ & $9-13$ & ---- & ----- \\
\hline TOTAL & $98(45+53)$ & $9-32$ & $30(10+20)$ & \\
\hline
\end{tabular}

Tabla 2. Aplicaciones realizadas para la evaluación del programa Tactus

En cuanto al programa Tactus empleado (del que ha habido una versión de prueba y otra definitiva, tal y como refleja la tabla de la muestra del estudio), éste ha sido descrito en anteriores publicaciones con detenimiento en cuanto al modelo didáctico y pedagógico, así como técnicamente (Tejada, Gil y García-Pérez, 2011; Morant, Gil, y Tejada, 2011). En el mismo, se hace uso de distintos audios y a través del teclado (mediante la barra espaciadora del ordenador) se marcan los pulsos y las figuras tratando de interpretar diferentes figuraciones rítmicas y lograr el mayor nivel de sincronización posible con la música escuchada. A continuación, se muestran pantallas capturadas del software utilizado.

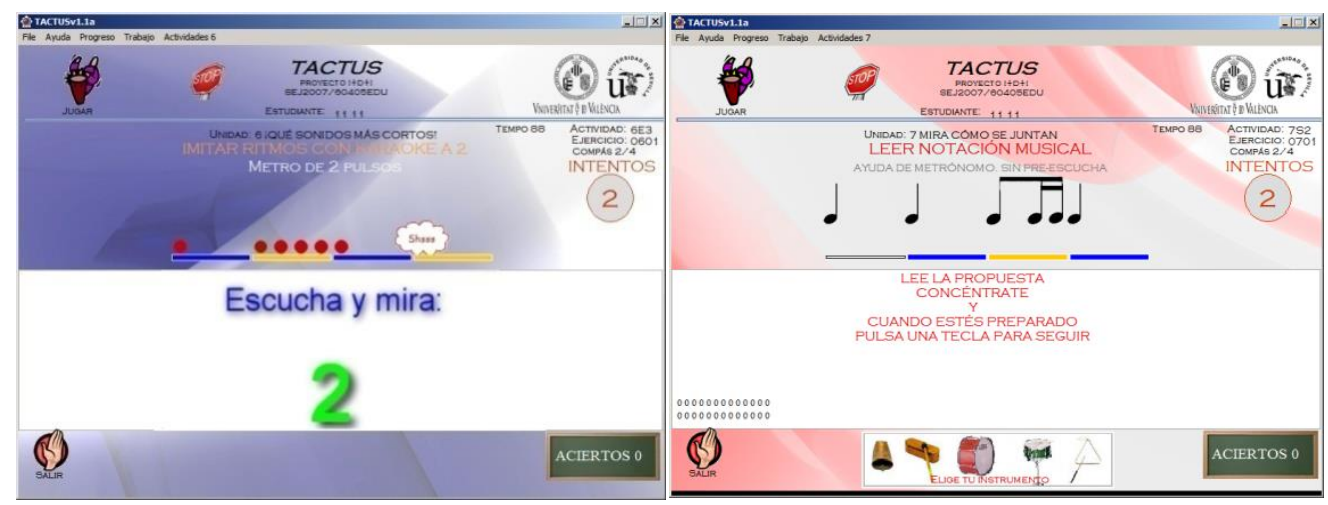

Figura 1. Pantallas principales del programa Tactus para el aprendizaje del ritmo musical

En el siguiente apartado, se procede a la descripción de las dimensiones y variables empleadas en el estudio.

\section{Dimensiones y variables}

\footnotetext{
${ }^{2} \mathrm{~h}=$ hombres; $\mathrm{m}=$ mujeres

${ }^{3}$ Centro con alumnos adultos matriculados en Licenciatura en Música y Licenciatura en Pedagogía Musical.

${ }^{4}$ Centro con alumnos adultos matriculados en Licenciatura en Música y Licenciatura en Pedagogía Musical.
} 
El análisis del discurso que se efectúa está basado en las aportaciones de Boza, Tirado \& GuzmánFranco (2010). Las categorías analíticas y la codificación utilizadas en el estudio incluyen las siguientes dimensiones y variables que se muestran a modo de resumen en la siguiente figura.

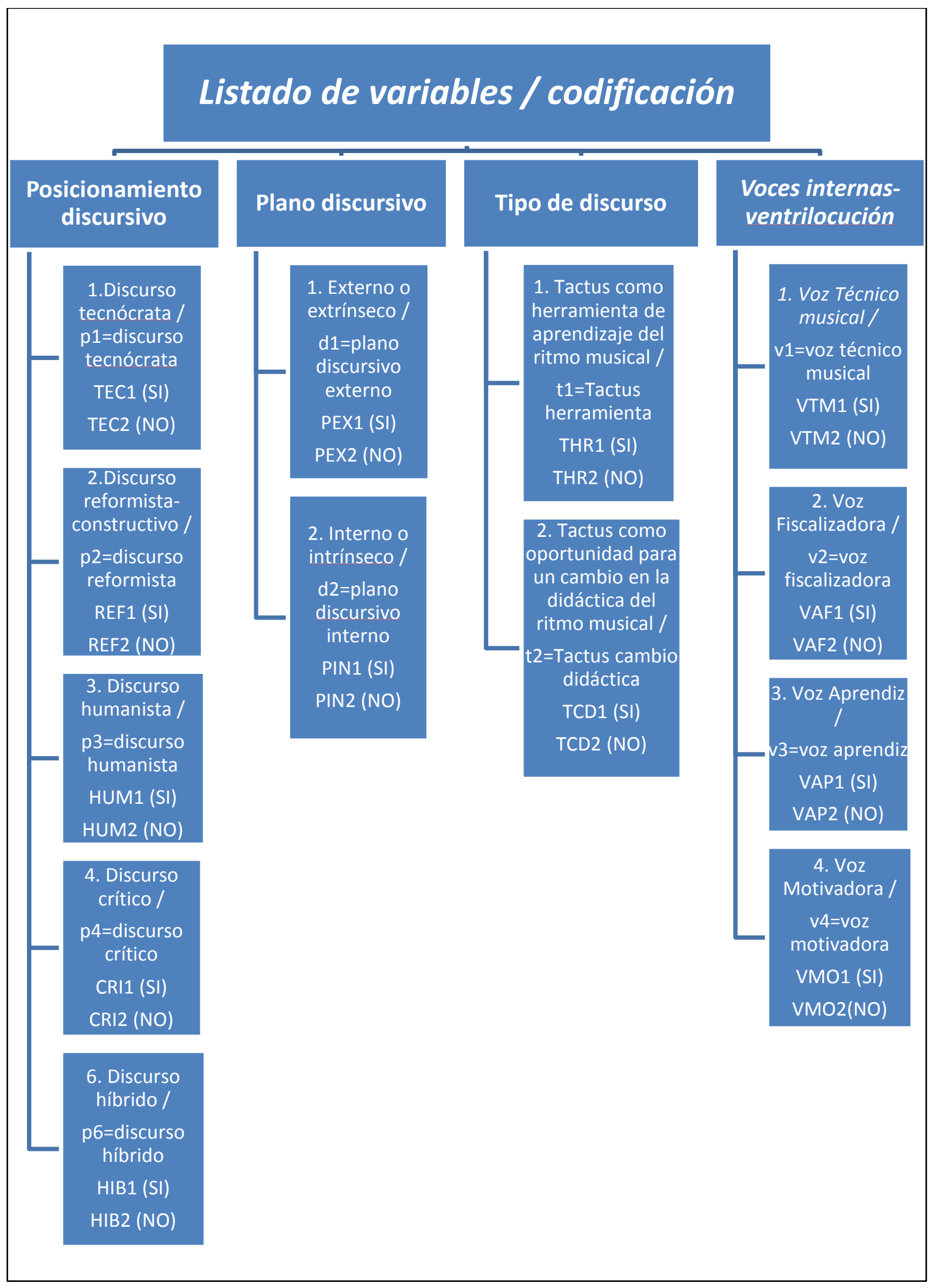

Figura 2. Categorías implicadas en el estudio

A continuación, se desarrollan los contenidos de la tabla anteriormente expuesta con un ejemplo de cada categoría, basado en los datos de las transcripciones del grupo de discusión: 
a) Posicionamientos discursivos (adoptados por el profesorado a la hora de valorar el software):

- Discurso tecnócrata: destaca las ventajas, posibilidades y beneficios del software para la didáctica del ritmo musical. Ejemplo: "A mí es que me podría servir mucho de refuerzo y además es una novedad en la clase" (fragmento 72).

- Discurso reformista-constructivo: destaca las ventajas del software, su posición es constructiva ya que la crítica reconoce las posibilidades del software y añade todas las posibles mejoras del mismo, a favor de una mayor efectividad y calidad para la didáctica del ritmo. Ejemplo: "Ahí, hay ejercicios que hay que tutelar, hay que tutelarlos. No se les puede dejar para que avancen porque se atrancan" (fragmento 14).

- Discurso humanista: emplea un discurso que manifiesta en la evaluación del software valores como el refuerzo positivo, la motivación o la importancia del compromiso con la ciudadanía activa o con los valores humanos. Ejemplo: "Lo más novedoso, [...] es que justamente se aprende música a través de un medio informal, que a los chiquillos ahora ya les resulta cercano, muchísimo más cercano en efecto que una partitura" (fragmento 90).

- Discurso crítico: destaca las desventajas del software, se emplean discursos orientados a hablar de aspectos negativos del software sin aportar mejoras, ideas o soluciones de las posibles deficiencias del mismo. Ejemplo: "yo lo que he encontrado es que en algunos momentos, sobre todo cuando había la notación no convencional, a veces lo que marcaba la pantalla, el ritmo con el teclado no era exacto" (fragmento 107).

- Discurso herético: destaca las desventajas del software de forma no-constructiva, incluso destructiva, el lenguaje incluso puede descalificar y rechazar el software y supone un planteamiento de resistencia, e incluso bloqueador de iniciativas innovadoras en el adiestramiento rítmico del alumnado. Ejemplo: "Se echa de menos la indicación del compás, que no aparece en ningún ejercicio, ni de creación, ni de subdivisión binaria ni ternaria" (fragmento 66).

- Discurso híbrido: mezcla de posicionamientos discursivos como tecnócrata-humanista en el que se emplean varios lenguajes que manifiestan la preferencia por el empleo de las TIC y los elementos emocionales implícitos en las mismas. Ejemplo: "A mí también me ha gustado el hincapié que hacen los ritmos ternarios y binarios tan continuo ¿no?" (fragmento 99).

b) Plano discursivo (indica si se habla desde un posicionamiento extrínseco o intrínseco al software):

- Externo o extrínseco: aspectos externos al docente y Tactus (acceso a la tecnología para el adiestramiento rítmico, disponibilidad de tiempo para emplear Tactus, apoyos recibidos para el aprendizaje del software educativo-musical, materiales empleados para la formación musical, formación del profesorado en TIC, referencias externas a Tactus o comparaciones con otros programas). Ejemplo: "Que si habéis pensado en una posibilidad que no salió aquí, que es tener un netbook en el cajón de la mesa de clase y sacarlo de vez en cuando para algún alumno que tenéis con problemas" (fragmento 41).

- Interno o intrínseco: aspectos internos del docente en su relación con Tactus (actitudes hacia Tactus, creencias sobre el software, dudas acerca del programa, prácticas con Tactus, resistencia a emplear Tactus). Ejemplo: "La memoria me gustó mucho, sobre todo por eso por... en educación auditiva lo trabajamos mucho y en este programa se trabaja bastante. Está muy bien" (fragmento 25).

c) Tipo de discurso sobre la naturaleza de la herramienta didáctica (valoración de la herramienta en sí o más allá de ella, el grado de trascendencia de la misma, como herramienta informática o como contribución para el cambio en la didáctica del ritmo):

- Tacuts como herramienta de aprendizaje del ritmo musical. Ejemplo: "Es una herramienta, la considero yo, por el simple hecho que uno puede decidir en realidad las actividades que les vamos a dar a los chiquillos y el progreso" (fragmento 8). 
- Tactus como oportunidad para un cambio en la didáctica del ritmo musical. Ejemplo: "la propuesta pedagógica para mí es altamente positiva en general, como idea general ¿no? después lo más alto así, lo más que me gustó [sic] fue lo que es el trabajo de la exactitud rítmica por ser un músico de percusión ¿no? ahí es que es lógico, lo de potenciar la memoria musical.. "(fragmento 87).

d) Voces internas-ventrilocución (privilegiación de contextos, voces y referentes del profesorado que emergen en la evaluación de Tactus):

- Voz Técnico musical: experto, especialista, información técnica y especializada. Ejemplo: "No, pero hay solo una pulsación. Lo digo por el ejercicio de Dalcroze. Tú le dices al niño, a dos, a tres, a cinco, a lo que quieras, pero lo que está escuchando el niño es una sola pulsación" (fragmento 50).

- Voz Fiscalizadora: persona que reprende lo malo que otros hacen, crítico con el programa. Ejemplo: "Entonces, no has visto que haya una evolución, una progresión metodológica, sino que te ha parecido que hay un salto demasiado brusco" (fragmento 71).

- Voz Aprendiz: se siente un poco ruborizado por el empleo de las TIC y habla desde la inseguridad y la duda. Ejemplo: "A ver... el tema del dictado; primero me costó un poco saber cómo funcionaba ipero esto es problema mío que no llego a más..." (fragmento 89).

- Voz Motivadora: es la voz del pedagogo musical que motiva y busca el refuerzo del alumnado en los procesos de aprendizaje rítmico con Tactus, su discurso se impregna de valores pedagógicos como la motivación o el refuerzo. Ejemplo: "Me parece muy importante lo accesible que es para los niños, dado que los chicos se manejan muy bien con el computador, es muy atractivo para ellos, [...] yo creo que es muy válido" (fragmento 18).

El análisis de los datos recogidos y codificados en el estudio aparece en el siguiente apartado.

\section{Análisis de datos}

En el proceso de análisis de los grupos de discusión, se diferencian y acotan distintos fragmentos discursivos $(n=115)$, a modo de unidad de análisis, en tanto que contienen elementos discursivos suficientes para ser interpretados en relación con el objetivo del estudio. Dichos fragmentos se detectan y codifican con el apoyo del programa de análisis cualitativo Atlas-ti. A la hora de codificar los datos recogidos, se ha seguido, además de un criterio semántico, un proceso inductivo y no emergente, es decir, se aplicó un proceso de codificación supeditado a la existencia previa de un sistema de categorías. De este modo y basados en la teoría (Boza, Tirado \& Guzmán-Franco, 2010), se han identificado y definido las categorías del estudio hasta su aplicación sistemática a los fragmentos (fragmento 1 a fragmento 115).

El resultado de la codificación se traduce en matrices de datos, tomando las categorías a modo de variables nominales dicotómicas (si-no). El programa SPSS permite un estudio descriptivo básico, entre otros aspectos. No obstante, el resultado principal del estudio proviene de la aplicación del programa $S P A D$ para elaborar un modelo de asociación basado en el análisis categórico multivariante, en que se aplican de forma encadenada las técnicas de correspondencias múltiples y clasificación (clustering), que han posibilitado reducir la descripción a 7 clases o tipologías discursivas utilizadas por el profesorado, que posteriormente quedan agrupadas en 5 clústeres (por su proximidad geométrica), mostrando con ello su posicionamiento e identidad ante la innovación.

A continuación, mostramos en el siguiente apartado los resultados del estudio.

\section{RESULTADOS}


Presentando, en primer lugar, los descriptivos básicos de las categorías discursivas estudiadas, se aprecia que éstas aparecen de forma variable en el total de los 115 fragmentos discursivos analizados, distribuyéndose como se muestra a continuación:

- Posicionamiento discursivo: el $27 \%$ de los casos $(n=31)$ aparece el posicionamiento discursivo tecnócrata, el 17,4\% reformista $(n=20)$, el 13\% humanista $(n=15)$, el 15,7\% crítico $(n=18)$, el $5,2 \%$ herético $(n=6)$, el $21,7 \%$ híbrido $(n=25)$.

- Plano discursivo: el plano externo del discurso supone el $17,4 \%(n=20)$, el $82,6 \%$ el plano interno $(n=95)$.

- Valoración discursiva: el discurso favorable a la consideración de Tactus como herramienta didáctica 51,3\% ( $n=59)$, como cambio en la didáctica del ritmo musical 48,7\% $(n=56)$.

- Voces: el discurso en el que aparece la voz interna de técnico musical representa el 53\% $(n=61)$, la voz fiscalizadora $7 \%(n=8)$, la voz de aprendiz $27 \%(n=31)$, la voz motivadora $13 \%(n=15)$.

A continuación, la Figura 2 muestra gráficamente las distribuciones comentadas.

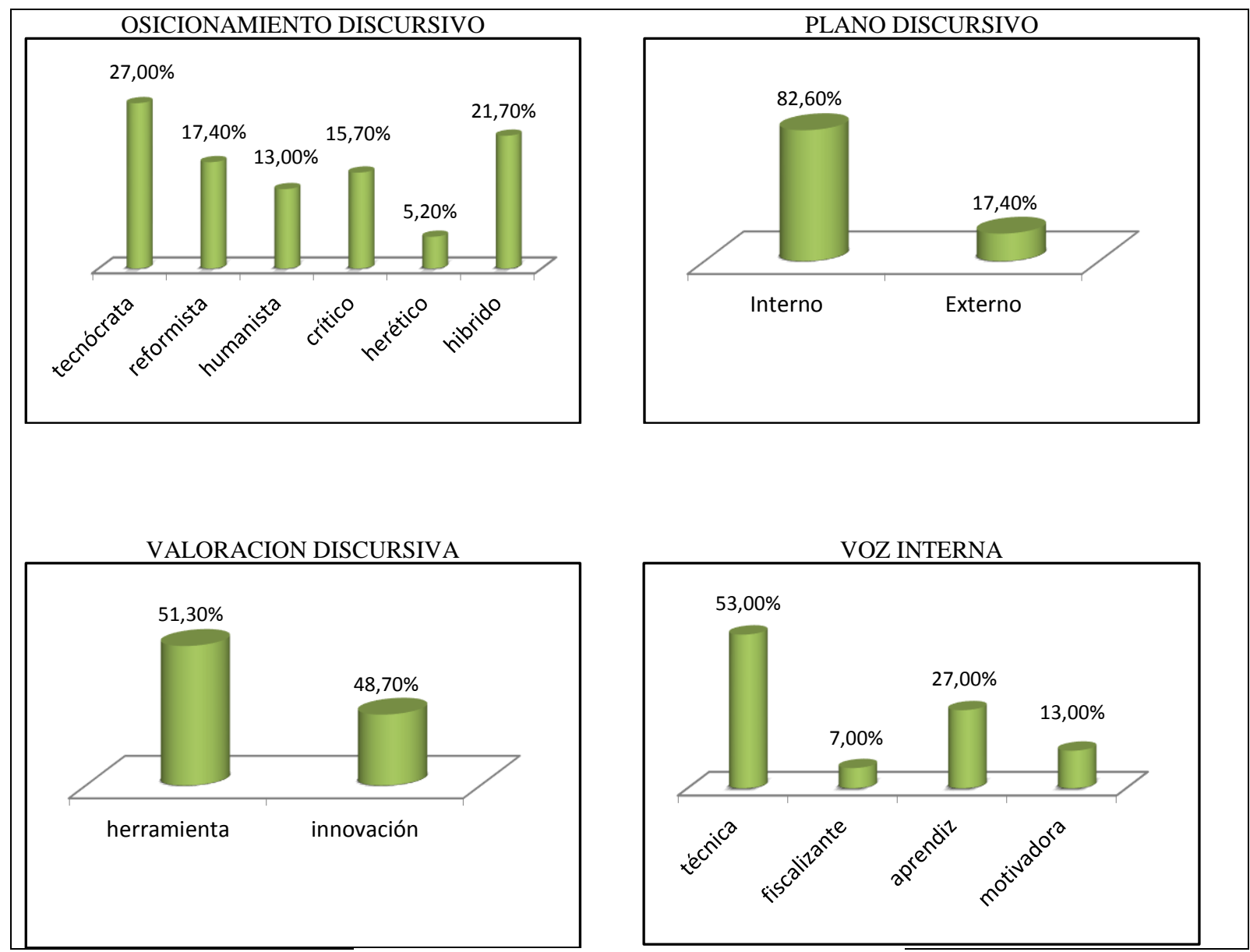

Figura 3. Distribución de las categorías del estudio

A partir de los resultados obtenidos se exploran los datos y se consigue elaborar un modelo categórico multivariante basado en las correspondencias múltiples entre las categorías incluidas para constituir el modelo estadístico de asociación. A la hora de construir el modelo, se han tenido en cuenta aquellas categorías que dentro de cada clase han supuesto un mayor grado significación y que aportaban mayor información a la hora de su elaboración. Las distintas categorías son: 


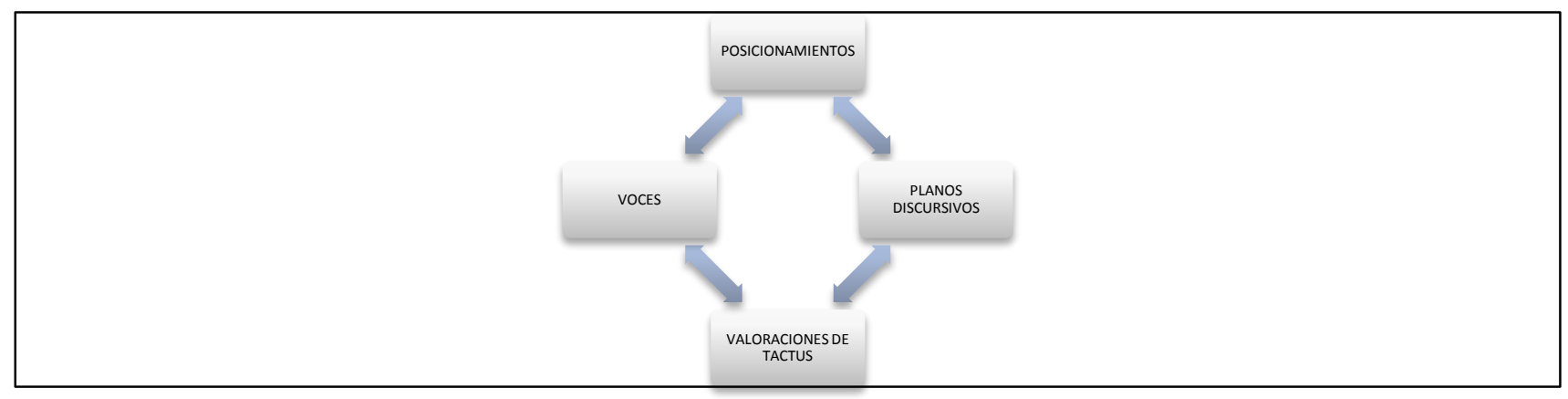

Figura. 4. Categorías que se han usado para la elaboración del modelo multivariante de 7 clases

Éste es el análisis de correspondencias múltiples, como paso previo a la realización del análisis clúster para la clasificación de los tipos de posicionamientos.

Se aprecia que 7 factores o componentes son suficientemente explicativos para explicar el 100\% de la tasa de inercia (al ser datos categóricos no hablamos de varianza), la siguiente tabla expone los resultados sobre el peso de cada categoría en los factores y los "cosinus carres" (grado de significación) que se interpretan como correlaciones con el factor que implican menor deformación de la perspectiva factorial al reducir las dimensiones y reflejar mejor cada categoría (C. carres > .300).

\begin{tabular}{|c|c|c|c|c|c|c|c|c|c|c|c|c|c|c|c|c|}
\hline \multicolumn{3}{|c|}{ MODALIDADES } & \multicolumn{7}{|c|}{ CONTRIBUCIONES } & \multicolumn{7}{|c|}{ COSINUS CARRES } \\
\hline IDEN/LIBELLE & P.REL. & DIS. & 1 & 2 & 3 & 4 & 5 & 6 & 7 & 1 & 2 & 3 & 4 & 5 & 6 & 7 \\
\hline \multicolumn{17}{|l|}{ Discurso Tecnócrata } \\
\hline TEC1 - TEC1 (SI) & 2.70 & 2.71 & 4.3 & 0 & 33.1 & 4.0 & 6.3 & 1.8 & 5.7 & .16 & 0 & .59 & .07 & .10 & .03 & .06 \\
\hline TEC2 - TEC2 (NO) & 7.30 & .37 & 1.6 & 0 & 12.2 & 1.5 & 2.3 & .7 & 2.1 & .16 & 0 & .59 & .07 & .10 & .03 & .06 \\
\hline \multicolumn{17}{|l|}{ Discurso Reformista } \\
\hline REF1 - REF1 (SI) & 1.74 & 4.75 & .5 & 2.7 & 1 & 50.3 & 3.8 & 7.5 & 2.2 & .02 & .06 & .02 & .74 & .05 & .10 & .02 \\
\hline REF2 - REF2 (NO) & 8.26 & .21 & .1. & .6 & 2 & 10.6 & .8 & 1.6 & .5 & .02 & .06 & .02 & .74 & .05 & .10 & .02 \\
\hline \multicolumn{17}{|l|}{ Discurso Humanista } \\
\hline HUM1 - HUM1 (SI) & 1.30 & 6.67 & .4 & 10.9 & 0 & 7.1 & 25.1 & 17.5 & 13.6 & .01 & .23 & 0 & .10 & .33 & .21 & .11 \\
\hline HUM2 - HUM2 (NO) & 8.70 & .15 & .1 & 1.6 & 0 & 1.1 & 3.8 & 2.6 & 2.0 & .01 & .23 & 0 & .10 & .33 & .21 & .11 \\
\hline \multicolumn{17}{|l|}{ Discurso Crítico } \\
\hline CRI1 - CRI1 (Si) & 1.57 & 5.39 & 5.8 & 3.0 & .6 & 8.0 & 29.4 & 5.0 & 18.8 & .19 & .06 & .01 & .12 & .40 & .06 & .16 \\
\hline CRI2 - CRI2 (NO) & 8.43 & .19 & 1.1 & .5 & .1 & 1.5 & 5.5 & .9 & 3.5 & .19 & .06 & .01 & .12 & .40 & .06 & .16 \\
\hline \multicolumn{17}{|l|}{ Discurso Herético } \\
\hline HER1 - HER1 (SÍ) & .52 & 18.17 & 3.6 & .1 & .4 & .4 & 13.0 & 51.5 & 19.9 & .10 & 0 & .01 & .01 & .16 & .57 & .15 \\
\hline HER2 - HER2 (NO) & 9.48 & .06 & .2 & 0 & 0 & 0 & .7 & 2.8 & 1.1 & .10 & 0 & .01 & .01 & .16 & .57 & .15 \\
\hline \multicolumn{17}{|l|}{ Discurso Híbrido } \\
\hline HIB1 - HIB1 (SÍ) & 2.17 & 3.60 & .7 & .3 & 40.4 & 12.0 & 4.7 & .9 & 2.8 & .02 & .01 & .67 & .19 & .07 & .01 & .03 \\
\hline HIB2 - HIB2 (NO) & 7.83 & .28 & .2 & .1 & 11.2 & 3.3 & 1.3 & .2 & .8 & .02 & .01 & .67 & .19 & .07 & .01 & .03 \\
\hline \multicolumn{17}{|l|}{ Plano externo } \\
\hline PEX1 - PEX1 (SÍ) & 1.74 & 4.75 & 11. & 25.2 & 7.3 & 0 & 1.2 & 2.6 & .2 & .38 & .56 & .01 & 0 & 02 & .03 & $\overline{0}$ \\
\hline PEX2 - PEX2 (NO) & 8.26 & .21 & 2.5 & 5.3 & .1 & 0 & .3. & .5 & 0 & .38 & .56 & .01 & 0 & 02 & .03 & 0 \\
\hline \multicolumn{17}{|l|}{ Plano interno } \\
\hline PIN1 - PIN1 (SÍ) & 8.26 & .21 & 2.5 & 5.3 & .1 & 0 & .3 & .5 & 0 & .38 & .56 & .01 & 0 & .02 & .03 & 0 \\
\hline PIN2 - PIN2 (NO) & 1.74 & 4.75 & 11.7 & 25.2 & .3 & 0 & 1.2 & 2.6 & .2 & .38 & .56 & .01 & 0 & .02 & .03 & 0 \\
\hline \multicolumn{17}{|l|}{ Tactus herramienta } \\
\hline THR1 - THR1 (SÍ) & 5.13 & .95 & 12.9 & 4.7 & 0 & 0 & 0 & .2 & 6.5 & .72 & .18 & 0 & 0 & 0 & 0 & .10 \\
\hline THR2 - THR2 (NO) & 4.87 & 1.05 & 13.6 & 4.9 & 0 & 0 & 0 & .2 & 6.8 & .72 & .18 & 0 & 0 & 0 & 0 & .10 \\
\hline \multicolumn{17}{|c|}{ Tactus cambio didáctica } \\
\hline TCD1 - TCD1 (SÍ) & 4.87 & 1.05 & 13.6 & 4.9 & 0 & 0 & 0 & .2 & 6.8 & .72 & .18 & 0 & 0 & 0 & 0 & .10 \\
\hline TCD2 - TCD2 (NO) & 5.13 & .95 & 12.9 & 4.7 & 0 & 0 & 0 & .2 & 6.5 & .72 & .18 & 0 & 0 & 0 & 0 & .10 \\
\hline
\end{tabular}

Tabla 4. Variables estudiadas, la contribución de cada una de las variables al modelo categórico multivariante y el grado de significación (cosinus carres) en cada eje

Como muestra la tabla anterior, el posicionamiento tecnócrata se refleja mejor en el eje 3, con un grado de contribución de 33.1 y un grado de significación de .59 . El posicionamiento reformista se refleja en el eje 4, con una contribución de 50.3 y un grado de significación de .74. El posicionamiento humanista se refleja en el eje 5, con un grado de contribución de 25.1 y un grado de significación de .33. El posicionamiento crítico se refleja en el eje 5, con un grado de contribución de 29.4 y un grado de significación de .40. El posicionamiento herético se refleja en el eje 6, con un grado de contribución de 51.5 y un grado de significación de .57 . El posicionamiento híbrido se refleja en el eje 3 , con un grado de contribución de 40.04 y un grado de significación de .67. La valoración de Tactus como herramienta se refleja en el eje 1, con un grado de contribución de 12.9 y un grado de significación de .72. La valoración de Tactus como cambio en la didáctica se refleja en el eje 1, con un grado de contribución de 13.6 y un grado de significación de .72. Una vez clarificado el modelo, con sus puntuaciones factoriales se elabora la partición en 7 clases (clustering). 
Como muestra el dendograma del modelo de 7 particiones construido (figura 5), se detecta un primer grupo formado por el $17 \%$ de la muestra, un segundo grupo formado con el $12 \%$, un tercero con el $20 \%$, un cuarto con el $17 \%$, un quinto con el $5 \%$ (más minoritario y con una menor presencia en el estudio, que además presenta una mezcla de diferentes modalidades en el discurso y posicionamientos, por lo que es el más heterogéneo de todos), un sexto con el $14 \%$ y un séptimo también con una presencia del $14 \%$.

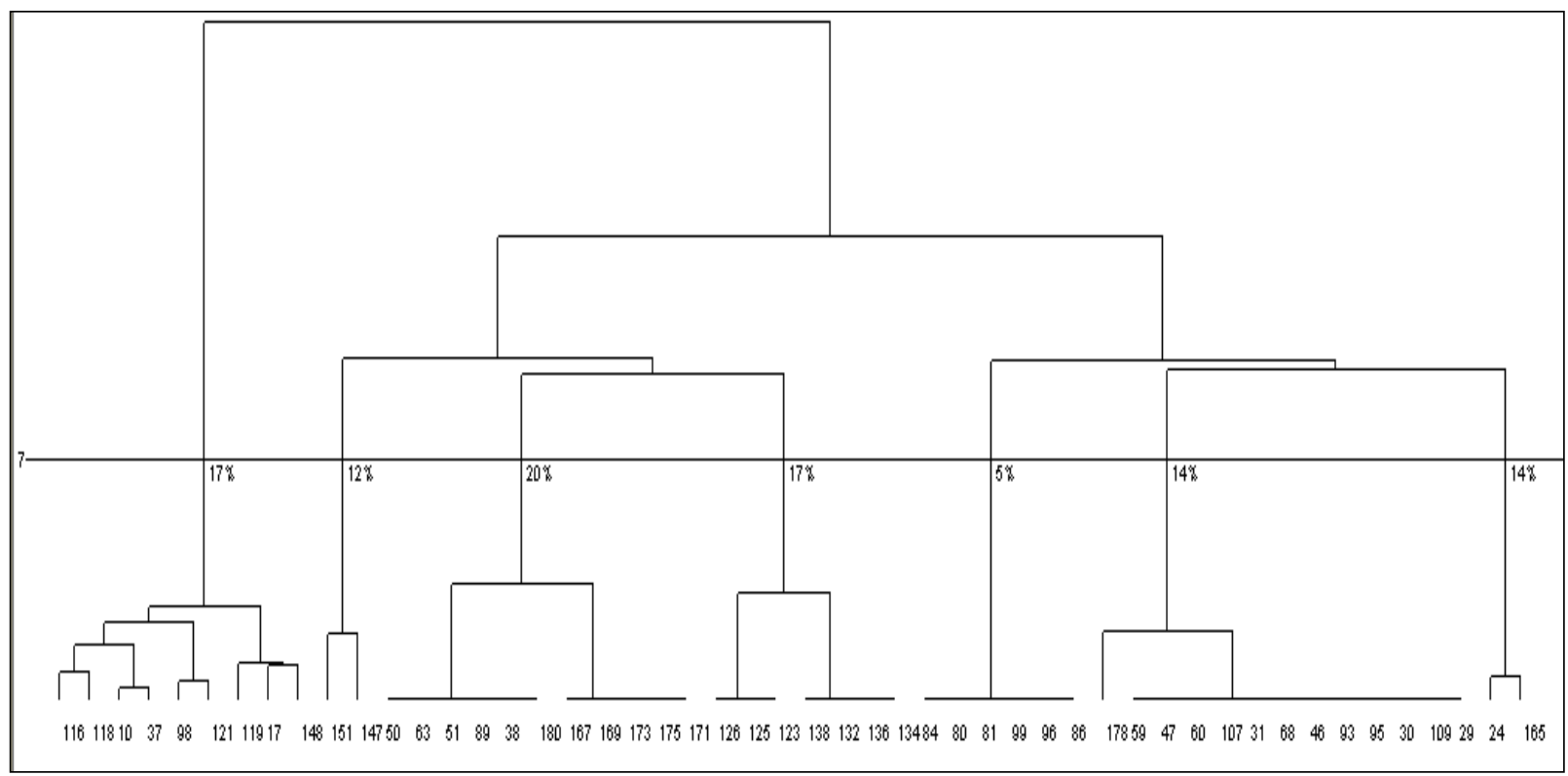

Figura 5. Dendograma del modelo categórico multivariante de 7 clases

A partir de los datos recogidos en el estudio se elabora un modelo de clasificación de 7 clases. Las 7 clases aparecen agrupadas en 5 clústeres. La proximidad geométrica de las distintas clases permite establecer 5 categorías o agrupamientos distintos, como se ve en el gráfico mostrado más adelante con el modelo elaborado en la investigación. Dichos clústeres se explican a continuación:

- El clúster 1 está formado por la primera clase, donde se han computado un total de 20 registros (17.39\% del total de registros de la muestra que es $n=115)$. La variable con mayor grado de significación en esta clase es "plano externo", que aparece representada por el $100 \%$ de los registros en dicha clase y representa el $100 \%$ de los registros de dicha clase en relación con el modelo elaborado. Asimismo, en esa primera clase, la "voz de técnico musical", supone el $53.04 \%$ del cómputo global de registros de la muestra, de ellos se han empleado el $80 \%$, lo que representa el $26.3 \%$ de la clase.

- El clúster 2 está representado por la segunda clase, donde se han computado un total de 14 registros (12.17\% del total de la muestra). La variable con mayor grado de significación es "discurso humanista", que está representada por el $100 \%$ de los registros y tiene un peso específico en esta clase del 93\%. Dentro del total de registros $(n=115)$ supone un $13.04 \%$. Además, la "voz motivadora" tiene una presencia del $13.04 \%$ en el cómputo total del modelo. De este porcentaje empleamos el $50 \%$ que supone un $46.67 \%$ de esta clase.

- El clúster 3 aparece en la tercera clase, donde se han computado un total de 23 registros (20\% del total de la muestra). La variable con mayor grado de significación es la correspondiente al "discurso tecnológico" (representada con un $26.96 \%$ en la totalidad de la muestra), aparece representada por el $100 \%$ de los registros de dicha clase y un peso del $74.19 \%$ en esta clase. Además, en esta clase, la variable "voz técnico musical", que está representada por el $78.26 \%$ de los registros tiene un peso específico en la clase del $29.51 \%$. También, en dicha clase, la variable "plano interno" está representada por el $82.61 \%$ de los registros. Se han computado el 100\% de dichos registros y tiene un peso del $24.21 \%$ en la clase.

- En el clúster 4 aparece la cuarta clase, donde se han computado un total de 20 registros del total de la muestra (lo que supone un $21.74 \%$ ). La variable con mayor grado de significación es el 
“posicionamiento híbrido". Se han empleado el 100\% de los registros y suponen un $80 \%$ en esta clase.

- El clúster 5 está representado por las clases 5, 6 y 7. En la quinta clase, se han computado un total de 6 registros $(5.22 \%$ del total de registros de la muestra). La variable con mayor grado de significación es el "posicionamiento herético" que representa un 5.22\% del total de registros de la muestra. Se emplearon el $100 \%$ de esos casos en la elaboración del modelo y tiene un presencia del $100 \%$ en esta clase. También, la voz de fiscal tiene una presencia del $6.96 \%$ del total de registros de la muestra. Se emplearon el $100 \%$ de los casos en la elaboración del modelo y tiene una presencia del $75 \%$ en esta clase. En la sexta clase, se han computado un total de 16 registros (13.91\% de los casos). La variable con mayor grado de significación es el "posicionamiento reformista" con un peso del $17.39 \%$ en el total de la muestra. Se han empleado el $100 \%$ de los casos y tienen en la clase un peso específico del $88.89 \%$. En la séptima clase, se han computado un total de 16 registros (13.91\% del total de la muestra). La variable con mayor peso en dicha clase es el "posicionamiento crítico", que supone el $15.65 \%$ del total de registros. Teniendo un peso específico en dicha clase del $88.89 \%$. Además, de la variable "valoración de Tactus como herramienta", que supone el $51.30 \%$ del total de registros, en esta clase se han computado $93.75 \%$, que tiene un peso específico en la clase 7 del $25.42 \%$.

A continuación, se muestra la figura 4 que expresa la distancia o cercanía de los cinco diferentes agrupamientos detectados y las categorías que los conforman a partir de las opiniones y discursos del profesorado que ha utilizado el software educativo. Como se observa en este modelo, dichas categorías (tal y como señalamos anteriormente) han sido establecidas a partir de la proximidad geométrica de las mismas estableciendo 5 agrupamientos distintos. El primer clúster del modelo aparece representado por el plano externo del discurso. El segundo clúster por el discurso humanista y la voz motivadora. El tercer clúster por el discurso tecnócrata. El cuarto por la hibridación discursiva. El quinto por el posicionamiento crítico, herético, reformista y la valoración del software empleado como herramienta para el aprendizaje del ritmo musical.

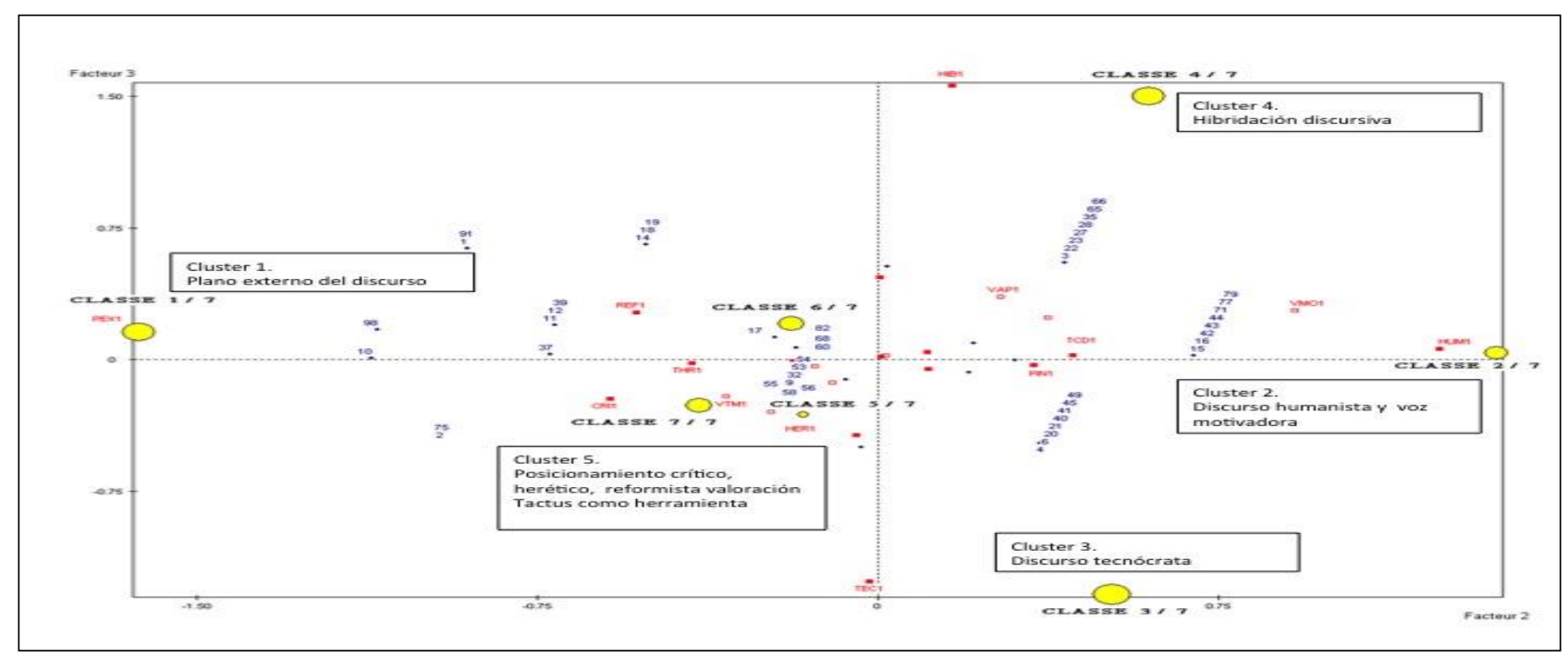

Figura 6. Modelo en el que se representan los 5 agrupamientos (clústeres) del discurso analizado en el modelo categórico multivariante 


\section{CONCLUSIONES Y PROSPECTIVAS DE LA INVESTIGACIÓN}

Las opiniones vertidas por el profesorado acerca del software Tactus aparecen en el modelo de manera polarizada, reflejadas por la distancia geométrica entre las mismas, representadas mediante diferentes clústeres o agrupamientos. Además, reflejan más allá de las categorías en las que se hallan ubicadas una actitud clara y definida en torno a la introducción de la innovación educativa en el adiestramiento rítmico. Dichas opiniones se resumen a continuación:

- En el primer clúster del modelo, se observa cómo el discurso del profesorado se caracteriza por la referencia a la implantación de las TIC, los problemas de accesibilidad a las nuevas tecnologías o la falta de recursos materiales, incluso la falta de formación que recibe el profesorado, además de la escasez de programas de adiestramiento rítmico parecidos a Tactus. Se denota un alto grado de carencia formativa en el profesorado en programas de Educación Musical y destaca la necesidad de una formación en este sentido. El profesorado acepta la utilidad de Tactus pero reconoce que está afectado por las limitaciones que se presentan en el acceso a las nuevas tecnologías.

- En el segundo clúster, destaca la hibridación discursiva, cuya distancia geométrica con respecto a otras variables plantea una categoría distinta y una dimensión diferente dentro del modelo elaborado. El profesorado, plantea un uso del lenguaje producto de la combinación de diferentes posicionamientos, como puede ser humanista y técnico al mismo tiempo o reformista y humanista.

- En el tercer clúster que constituye el modelo, se ve la proximidad geométrica entre el posicionamiento humanista y la voz motivadora. Tactus, tal y como se observa en el discurso suscita emociones en el profesorado, como muestran las valoraciones vertidas en los grupos de discusión. El empleo del discurso humanista posiciona a los sujetos en una valoración o evaluación de Tactus en la que están presentes las emociones tanto positivas como negativas suscitadas por su uso, las cuales suelen venir acompañadas de un discurso de apoyo a Tactus desde un posicionamiento más tecnócrata o más crítico, dependiendo del grado de satisfacción experimentada en el empleo del software para el aprendizaje del ritmo. Este discurso humanista aparece vinculado a opiniones en las que el profesorado manifiesta su entusiasmo. Además, destaca la importancia de Tactus como cambio en la didáctica y la motivación que provoca en el alumnado, ofreciendo una nueva manera de trabajar el ritmo y promover emociones positivas en torno al aprendizaje musical.

- En el cuarto clúster, destaca la presencia del discurso tecnócrata. Este discurso señala la importancia de la implantación de las nuevas tecnologías en la Educación Musical y plantea la necesidad de un mayor grado de uso de las mismas. El empleo de Tactus despierta el interés en el profesorado, que observa las posibilidades de Tactus para el aprendizaje del ritmo musical. Resalta la novedad del software y sus prestaciones para la didáctica de la música y el lenguaje musical, competencias básicas e imprescindibles en la formación del futuro músico.

- En el quinto clúster, en el que se agrupan la quinta, sexta y séptima clase, aparecen destacados los discursos herético, crítico y reformista y el empleo de Tactus como herramienta. Esta dimensión plantea una cercanía entre la valoración negativa del programa Tactus y la consideración de Tactus como una herramienta que requiere ser transformada para dar cabida a las exigencias de un software de formación musical. Por lo tanto, no reconoce la posibilidad de Tactus como un cambio en la didáctica del ritmo musical y sus posibilidades en este sentido. En este grupo se engloban las críticas no constructivas e incluso destructivas, que no aportan soluciones a las carencias apreciadas en Tactus y por lo tanto un planteamiento tecnófobo de la Educación Musical, viendo sólo el lado negativo de la tecnología y sus deficiencias, sin observar la validez, actualidad y necesidad de la implantación de nuevos recursos necesarios para la mejora de la calidad en la Educación Musical y la formación tanto del profesorado como el alumnado.

El trabajo muestra que mediante el análisis de los discursos del profesorado en la evaluación de software educativo aparecen posturas diversas. Las posturas legitimadoras (tecnócratas) de las TIC en Educación Musical promueven contextos de aprendizajes mediados por las nuevas tecnologías y potencian el desarrollo del aprendizaje musical con software específico como Tactus, para el 
aprendizaje del ritmo musical, elemento esencial y competencia básica en la formación de cualquier músico.

Las opiniones contrarias a la formación musical con Tactus, denotan una falta de interés e incluso bloqueo del desarrollo del sentido del ritmo con nuevas herramientas de aprendizaje basadas en las TIC. Éstas quedan de manifiesto en los posicionamientos crítico o herético, contrarios al cambio en las formas tradicionales de aprendizaje del ritmo musical.

Las opiniones a favor de la formación musical con Tactus, muestran que la mayor parte del profesorado manifiesta en sus discursos una actitud abiertamente positiva a la introducción de este tipo de herramientas reflejadas en un discurso tecnócrata, voces humanistas y actitud motivadora. Esto denota el apoyo al uso de software de adiestramiento rítmico y su introducción en los métodos de aprendizaje musical tradicionales. Las opiniones y discursos negativos del profesorado, de oposición, bloqueo o resistencia al cambio son significativamente reducidos y se observa la preferencia por un mayor uso de las TIC en la Educación Musical.

En definitiva, las voces, posicionamientos, discursos y opiniones del profesorado en torno al software empleado constituyen una aportación a la investigación educativa y son un instrumento de gran valor para observar las perspectivas, puntos de vista y dimensiones acerca de la evaluación cualitativa de dicho software. El modelo clúster elaborado a partir de estos discursos docentes nos permite valorar de manera estructurada y objetiva las opiniones en torno al software para el adiestramiento rítmico. Además, ofrece diferentes perspectivas y aporta distintas aproximaciones a la evaluación del software educativo, permitiendo así una visión más rica, completa y a la vez profunda del mismo. Todo ello, contribuye a la toma de decisiones educativas para la mejora y optimización de su rendimiento, calidad y efectividad como mediador en los procesos de enseñanzaaprendizaje del ritmo musical.

Entre las limitaciones del estudio, destaca la necesidad de contar con una muestra más numerosa y homogénea entre profesorado participante y los diferentes perfiles profesionales del mismo. Por otro lado, las edades y características madurativas y psicoevolutivas del alumnado participante en el uso del software también podrían ser más homogéneas, lo cual permitiría en las percepciones del profesorado una visión más precisa y objetiva acerca del software evaluado.

Como prospectiva de la investigación y de cara a enriquecer la información aportada en este trabajo, se plantea la posibilidad de hacer otros estudios que atiendan a la percepción del alumnado que emplea el software para el adiestramiento rítmico. Otros estudios que triangulen lo que el profesor manifiesta en sus opiniones y discursos y lo que hace realmente hace en el aula serían susceptibles de constituir futuras investigaciones. También se plantea el desarrollo del análisis del discurso en la evaluación del software para la Educación Musical como línea de investigación en el futuro, tomando como base las categorías establecidas en el estudio de Boza et al. (2010) que se han ampliado y adaptado al presente trabajo. Además de incorporar dichas categorías al estudio de software para el adiestramiento rítmico como el planteado aquí y en otras investigaciones como la de Tudurí et al. (2013) para la obtención de nuevos modelos estadísticos que optimicen su uso y eficacia. Todo ello, orientado a una mejor detección de necesidades y carencias en los contextos formativos mediados por las tecnologías de la información y la comunicación, en el marco de la actual sociedad del conocimiento. En definitiva, el trabajo ofrece una manera estructurada de obtener información del rendimiento, características y posibilidades del software para el adiestramiento rítmico. Todo ello a partir del análisis de las opiniones y los discursos docentes en torno a las nuevas tecnologías aplicadas al desarrollo de competencias y destrezas rítmicas en el alumnado de Educación Musical.

\section{Referencias}

Alonso, C., Casablancas, S., Domingo, L., Guitert, M., Moltó, O., Sánchez i Valero, J. A., \& Sancho, J. M. (2010). De las propuestas de la administración a las prácticas en el aula. Revista de 
Educación, 352, 53-76. Consultado en http://www.revistaeducacion.educacion.es/ re352/re352.pdf

Aviram, R. (2002, 26-28 de junio). ¿Conseguirá la educación domesticar a las TIC? Ponencia presentada en el II Congreso Europeo de Tecnologías de la Información en la Educación y la Ciudadanía: Una Visión Crítica. Barcelona.

Bolívar, A., Fernández Cruz, M., \& Molina, E. (2004). Investigar la identidad profesional del profesorado: Una triangulación secuencial. Forum: Qualitative Social Research, 6(1). Consultado en http://nbn-resolving.de/urn:nbn:de:0114-fqs0501125 el 10/9/2014.

Bolívar, A. (2007). La formación inicial del profesorado de secundaria y su identidad profesional. Estudios sobre Educación, 12, 13-30. Consultado en http://dspace.si.unav.es/dspace/ bitstream/10171/8987/1/12\%20Estudios\%20Ea.pdf

Boza, A., Tirado, R., \& Guzmán-Franco, M. D. (2010). Creencias del profesorado sobre el significado de la tecnología en la enseñanza: influencia para su inserción en los centros docentes andaluces. Revista Electrónica de Investigación y Evaluación Educativa, 16(1), 1-24. Consultado en http://www.uv.es/RELIEVE/v16n1/RELIEVEv16n1_5.htm

Brickner, D. L. (1995). The effects of first and second order barriers to change on the degree and nature of computer usage of mathematics teachers: A case study. Dissertation Abstracts International, 56(1), 07A. (UMI No. 9824700). Consultado en http://dl.acm.org/ citation.cfm?id=922086 el 1/10/2014.

Coll, C., \& Falsafi, L. (2010). Learner identity. An educational and analytical tool. Revista de Educación, 353, 211-233. Consultado en http://www.revistaeducacion.educacion.es/ re353.htm el 3/9/2014.

Davies, B., \& Harré R. (1990). Positioning: The discursive production of selves. Journal for the Theory of Social Behaviour, 20(1), 44-63.

De Pablos, J. (1999). Las nuevas tecnologías y la construcción de la identidad cultural. El cambio educativo para el siglo XXI. Bordón, 51(4), 417-433.

Dubar, C. (2002). La crisis de identidades. La interpretación de una mutación. Barcelona: Bellaterra.

Ertmer, P. A. (1999). Addressing first and second-order barriers to change: Strategies for technology integration. Educational Technology Research and Development, 47(4), 47-61. Consultado en http://link.springer.com/journal/11423 el 2/8/2014

Harré, R. \& Langenhove, L. (1999). Positioning Theory: Moral Contexts of Intentional Action. Oxford: Blackwell.

Georgii-Hemming, E., \& Westvall, M. (2010): Teaching music in our time: student music teachers' reflections on music education, teacher education and becoming a teacher. Music Education Research, 12(4), 353-367.

Konstantidinis, A., Thrasyvoulos, T., Theodouli, T., \& Pomportsis, A. (2010). Fostering collaborative learning in Second Life: Metaphors and affordances. Computers \& Education, 55, 603-615. Consultado en http://www.journals.elsevier.com/computers-and-education el 6/10/2014.

Morant, R., Gil, M. P., \& Tejada, J., (2011) Tactus, un programa informático para el adiestramiento y mejora rítmica en la red de Escuelas de Música de la Federación (XEMFE). Música i Poble, $165,32-33$.

Partti, H., \& Karlsen, S. (2010): Reconceptualising musical learning: new media, identity and community in music education. Music Education Research, 12(4), 369-382.

Pelgrum, W. J. (2001). Obstacles to the integration of ICT in education: Results from a worldwide educational assessment. Computers \& Education, 37(2), 163-178. 
Rebollo, M. A. (2001). Discurso y educación. Sevilla: Mergablum.

Rebollo, M. A. (2002). Estudio y caracterización del discurso escolar: Aplicación del Análisis de Correspondencias Múltiples. Revista de Investigación Educativa, 20(1), 131-151. Consultado en http://revistas.um.es/rie/article/viewFile/97541/93611

Rebollo, M. A. \& Hornillo, I. (2010). La perspectiva emocional en la construcción de la identidad en contextos educativos: discursos y conflictos emocionales. Revista de Educación, 353, 235263. Consultado en http://www.revistaeducacion.educacion.es/re353/re353_09.pdf

Reynolds, R., Walker, K., \& Speight C. (2010). Web-based museum trails on PDAs for universitylevel design students: Design and evaluation. Computers \& Education, 55, 994-1003.

Smith, M. (1988). Developing youth work: Informal education, mutual aid and popular practice. Milton Keynes: Open University Press.

Tejada, J., Gil, M. P., \& García-Pérez, R. (2011) Tactus: Didactic design and implementation of a pedagogically sound based rhythm-training computer program. Journal of Music, Technology and Education, 3(2-3), 155-167. Consultado en http://www.ingentaconnect. com/ content/intellect/jmte/2011/00000003/f0020002/art00005 el 1/9/2014.

Tiernan, P. (2010). Enhancing the learning experience of undergraduate technology students with LabVIEW software. Computers \& Education, 55, 1579-1588.

Triantafyllaki, A. (2010). Performance teachers' identity and professional knowledge in advanced music teaching. Music Education Research, 12:1, 71-87.

Tudurí, A., Serra, B. \& Mut, B. (2013). Objective rhythmic performance evaluation tool (O.R.P.E.T.): A numerical method to evaluate the accuracy of a musical performance. Journal of Music, Technology \& Education, 6(1), 61-80. Consultado en http://www.ingentaconnect.com/content/intellect/jmte/2013/00000006/00000001/art00005 el 3/9/2014.

Wertsch, J. (1993). Voces de la mente. Madrid: Visor.

Manuscrito recibido el 28/09/2013 y evaluado anónimamente. Aceptado para su publicación el 04/06/2014. 